\title{
Improved Effective TMD Factorization for forward dijet production in p-A Collisions
}

\author{
Piotr Kotko ${ }^{* \dagger}$ \\ Department of Physics, The Pennsylvania State University, University Park, PA 16802, USA \\ E-mail: pxk19epsu.edu
}

\begin{abstract}
We propose a new factorization formula for forward dijet production in dilute-dense collisions which encompasses both the saturation regime and the dijet decorrelation regime. The first regime is inherited from the back-to-back correlation limit of the Color Glass Condensate framework, while the other is specific to the High Energy ( or $k_{T}$ ) Factorization. The new formula is an extrapolation of the Transverse Momentum Dependent (TMD) factorization approach to the case when the off-shellness of the incoming small- $x$ gluon is not neglected. The off-shell gauge invariant hard factors corresponding to various TMD gluon distributions are calculated in two independent ways, in particular using color decomposition and helicty method.
\end{abstract}

XXIII International Workshop on Deep-Inelastic Scattering,

27 April - May 12015

Dallas, Texas

\footnotetext{
* Speaker.

${ }^{\dagger}$ In collaboration with A. van Hameren, K. Kutak, C. Marquet, E. Petreska, S. Sapeta.
} 


\section{Introduction}

In the present contribution we discuss proton-nucleus collisions at LHC energies, where the observed final states consist in a dijet system produced in the forward direction (relative to proton) with relatively large transverse momentum of individual jets. In the LO partonic picture of the collision the incoming partons have to carry a highly asymmetric longitudinal fractions $x$ of the incoming hadrons, $x_{A} \ll x_{B}$, where $x_{B}$ is the fraction of the proton momentum $p_{B}$ and $x_{A}$ is the fraction of the nucleus momentum $p_{A}$ (see Fig. 1 below). We assume that the nucleus is probed at small $x_{A}$ where parton saturation phenomenon [1] enters the game and the collinear factorization is insufficient to address the problem. One of the suitable approaches is the Color Glass Condensate (CGC) [3], in particular so-called hybrid approach [2] which assumes the dilute-dense collisions with the kinematics outlined above. In this approach, the large- $x$ parton is treated within the standard collinear factorization while the multiple interactions are attributed to the nucleus only. Let us note, that the CGC approach, in general, differs from the 'standard' factorization picture. Namely, the cross section is expressed in terms of wave functions propagating through color glass field described in terms of various correlators of Wilson lines, which in principle can be obtained from models, but are not universal. Despite the complexity, the CGC approach has been successfully applied to the phenomenology at RHIC $[4,5,6]$. At LHC, however, the typical transverse momenta of produced jets are much larger. On one hand, the presence of an additional large scale may simplify the approach leading to an 'effective' factorization, but on the other hand may also introduce another sources of 'large logs'. In the next section we shall discuss available approaches in connection to the relative magnitude of the scales present in the process under consideration.

\section{Scale regimes}

The process under consideration is depicted in Fig. 1. The jets are produced with certain average transverse momentum of the order of $P_{T}$ which by definition sets the largest scale in our problem. Due to the emissions which are not tagged the jets are decorrelated by an amount $k_{T}=$ $\left|\vec{k}_{T}\right|=\left|\vec{p}_{T 1}+\vec{p}_{T 2}\right|$ where $\vec{p}_{T 1}, \vec{p}_{T 2}$ are transverse momenta of the jets. In the spirit of the hybrid formalism, the momentum transfer $\vec{k}_{T}$ enters the nucleus nonperturbative part. This measurable quantity sets another, possibly large, scale in our problem. Finally, the third scale is given by the saturation scale $Q_{s}$ which increases with $x_{A}$ decreasing. When all three scales are of the same order $Q_{s} \sim k_{T} \sim P_{T}$ the CGC formalism is the best-suited approach. However, as we have already indicated, at LHC the jets are produced with relatively large transverse momentum. In general the situation is quite compex; below we list the scale regimes depending on the relative magnitude of the three scales involved:

- Collinear Factorization. Suppose, for the illustration, that $x_{A}$ is not significantly small so we are outside the saturation regime, $Q_{s} \sim \Lambda_{\mathrm{QCD}}$ and the jets are produced back-to-back, $k_{T} \ll P_{T}$. Then, essentially we are in the standard leading twist collinear factorization regime, where the cross section is expressed by the convolution of collinear (or 'integrated') PDFs and on-shell amplitude squared.

- High Energy Factorization (HEF). If $x_{A}$ is small but $Q_{s} \ll k_{T} \sim P_{T}$ we are outside the saturation regime, but the BFKL-type resummation [7] is needed. This leads to the High Energy 


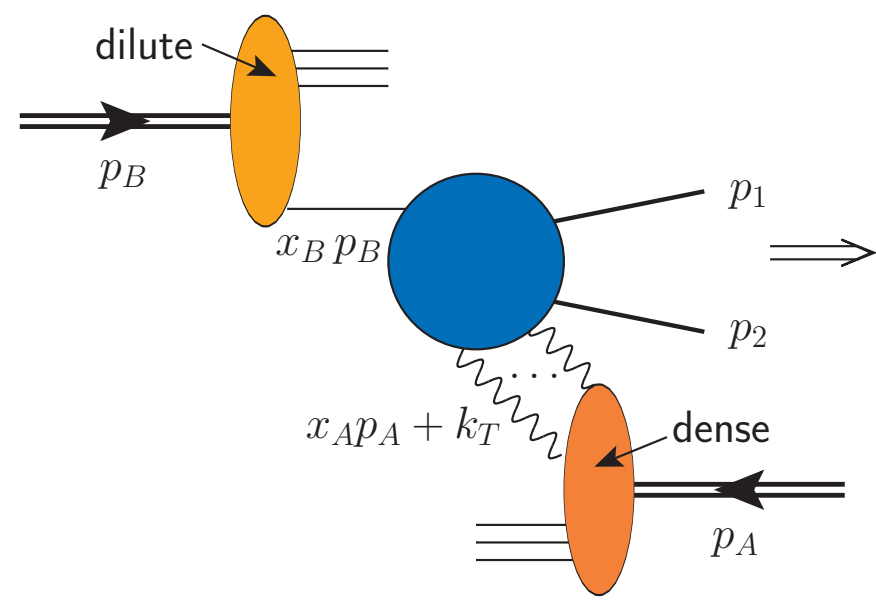

Figure 1: A partonic interpretation of forward dijet production in the dilute-dense collision within the hybrid factorization approach. The upper parton entering the partonic sub-process is treated within the collinear factorization. The parton (or multiple partons) entering the nucleus nonperturbative part carries a transverse momentum.

or $k_{T}$-factorization $[1,8,9,10]$ where the cross section is expressed in terms of Unintegrated Gluon Distribution (UGD) (for nucleus) and off-shell gauge invariant amplitude squared. Since the $k_{T}$ is not neglected in both the non-perturbative part and the hard sub-process the HEF approach takes into account higher twist contributions. This approach has been recently applied to describe some LHC jet data $[11,12,13]$ with however an additional Sudakov-type resummation needed whenever $k_{T} \ll P_{T}$ i.e. when jets are produced back-to-back. This resummation is 'external' with respect to the standard HEF and is included by the hard scale dependence of UGDs.

- Generalized TMD Factorization. When $k_{T} \sim Q_{s} \ll P_{T}$ one can establish an effective factorization [14]. Namely, using the CGC approach in that limit one can show that at large $N_{c}$ the cross section involves two distinct UGDs: so-called 'dipole' UGD $G_{2}$ and the WeizsackerWilliams UGD $G_{1}$. These UGDs are universal, i.e. they can be accessed in different processes. It turns out that these two UGDs can be recognized also when constructing the Transverse Momentum Dependent (TMD) gluon distributions using [15] and taking the large $N_{c}$ and small $x$ limits [14] (actually the small $x$ limit is taken into account by additional CGCmotivated assumptions). This connection provides also the operator definitions for UGDs.

The last approach is very attractive as it provides a tool to describe the process under consideration in terms of factorization. The main limitation is that it is restricted to the back-to-back correlation region of the phase space. Though the main contribution to the cross section comes indeed from that region, the data reveals large decorrelations of the jets (see e.g. [16, 17]). Thus, the possible extension to the region $k_{T} \sim P_{T}$ is highly desirable. This has been done in [18] and below we shall describe this improved approach. 


\section{Improved TMD Factorization}

Before we present our main result, let us recall how the TMD approach works. Suppose we want to find a TMD gluon distribution for a given partonic process, say $q g \rightarrow q g$. As in case of any gluon distribution, the primordial definition is proportional to the Fourier transform of a matrix element of a nonlocal operator $\left\langle P\left|\operatorname{Tr} F^{+i}(\xi)[\xi, 0] F^{+i}(0)\right| P>\right.$, where $F$ are gluon field-strength tensors in the fundamental representation with an appropriate spin projection, $[\xi, 0]$ is a Wilson link in the fundamental representation required for a gauge invariance of the non-local operator and $|P\rangle$ is a hadronic state. Unlike in the collinear factorization, the fields are separated in the transverse direction, in addition to the light-cone separation. The precise form of the gauge links depends on the process under consideration. This is because it is the resummation of collinear gluons (collinear to the incoming gluon) what determines the gauge links. Each external leg contributes a Wilson line, which are then 'glued' by the color structure of the pertinent diagram. Thus, different color flows will have different gauge links and thus different TMD gluon distributions [15]. In principle, this means that the universality is lost. However, as already mentioned in the previous section, in [14] it was shown that the universality is recovered at the large $N_{c}$ limit, provided some additional CGC-motivated assumptions are made.

In the above discussion it was assumed that all the external lines were on-shell, including the line for which the TMD gluon densities were constructed. This corresponds to the back-to-back correlation limit $k_{T} \ll P_{T}$, i.e. the off-shellnes $k_{T}$ is neglected in the hard process, but not in the gluon TMD distributions. The crucial observation is that when the off-shellness is kept for the leg for which we construct the TMD distribution, in certain gauge the diagrammatic content of the hard process can be preserved preserving also the gauge invariance. This can be seen by first constructing the gauge invariant off-shell amplitudes using e.g. [19, 20, 21] (corresponding also to the Lipatov's effective vertices [22]), and next choosing the appropriate gauge to get rid of 'eikonal' diagrams.

Our result can be summarized by the following factorization formula

$$
\frac{d \sigma^{p A \rightarrow \text { dijets }+X}}{d^{2} P_{T} d^{2} k_{T} d y_{1} d y_{2}}=\frac{\alpha_{s}^{2}(\mu)}{\left(x_{A} x_{B} S\right)^{2}} \sum_{a, c, d} x_{B} f_{a}\left(x_{B}, \mu\right) \sum_{i=1}^{2} K_{a g^{*} \rightarrow c d}^{(i)} \Phi_{a g \rightarrow c d}^{(i)}\left(x_{A}, k_{T}, \mu\right) \frac{1}{1+\delta_{c d}},
$$

where $y_{1}, y_{2}$ are the rapidities of the outgoing parton momenta $p_{1}, p_{2}, P_{T}=(1-z) p_{T 1}-z p_{T 2}$ with $z=p_{1}^{+} /\left(p_{1}^{+}+p_{2}^{+}\right)$. Further, $f_{a}$ is the collinear PDF for parton $a, \Phi_{a g \rightarrow c d}^{(i)}, i=1,2$ are the TMD gluon distributions corresponding to subprocess $a g \rightarrow c d$, and, finally, $K_{a g^{*} \rightarrow c d}^{(i)}, i=1,2$ are the pertinent hard factors calculated from the gauge invariant color-ordered amplitudes corresponding to $a g^{*} \rightarrow c d$ subprocess. Let us mention that the color-decomposition technique [23] has proved to be very useful here. Namely, we have re-derived the TMD gluon distributions for $2 \rightarrow 2$ processes of [15] using color decomposition instead of Feynman diagrams and found that there are only two independent TMD gluon distributions per channel. Moreover, the color-ordered amplitudes have some nice properties: they are gauge invariant (even for the off-shell case), there are just few of them, and all the hard factors $K^{(i)}$ can be easily constructed in terms of suitable combinations of the color-ordered amplitudes. For more details we refer to our original work [18].

The expressions for the TMD gluon distributions at finite $N_{c}$ and for the hard factors are listed in the box below: 


$$
\begin{aligned}
& K_{g q \rightarrow g q}^{(1)}=-\frac{\bar{u}\left(\bar{s}^{2}+\bar{u}^{2}\right)}{2 \bar{t} \hat{t} \hat{s}}\left(1+\frac{\bar{s} \hat{s}-\bar{t} \hat{t}}{N_{c}^{2} \bar{u} \hat{u}}\right) \\
& \Phi_{g q \rightarrow g q}^{(1)}=\mathscr{F}_{q g}^{(1)} \\
& K_{g q \rightarrow g q}^{(2)}=-\frac{C_{F}}{N_{c}} \frac{\bar{s}\left(\bar{s}^{2}+\bar{u}^{2}\right)}{\bar{t} \hat{t} \hat{u}} \\
& \Phi_{g q \rightarrow g q}^{(2)}=\frac{1}{N_{c}^{2}-1}\left(-\mathscr{F}_{q g}^{(1)}+N_{c}^{2} \mathscr{F}_{q g}^{(2)}\right) \\
& K_{g g \rightarrow q \bar{q}}^{(1)}=\frac{1}{2 N_{c}} \frac{\left(\bar{t}^{2}+\bar{u}^{2}\right)(\bar{u} \hat{u}+\bar{t} \hat{t})}{\bar{s} \hat{s} \hat{t} \hat{u}} \\
& \Phi_{g g \rightarrow q \bar{q}}^{(1)}=\frac{1}{N_{c}^{2}-1}\left(N_{c}^{2} \mathscr{F}_{g g}^{(1)}-\mathscr{F}_{g g}^{(3)}\right) \\
& K_{g g \rightarrow q \bar{q}}^{(2)}=\frac{1}{4 N_{c}^{2} C_{F}} \frac{\left(\bar{t}^{2}+\bar{u}^{2}\right)(\bar{u} \hat{u}+\bar{t} \hat{t}-\bar{s} \hat{s})}{\bar{s} \hat{s} \hat{t} \hat{u}} \\
& \Phi_{g g \rightarrow q \bar{q}}^{(2)}=-N_{c}^{2} \mathscr{F}_{g g}^{(2)}+\mathscr{F}_{g g}^{(3)} \\
& K_{g g \rightarrow g g}^{(1)}=\frac{N_{c}}{C_{F}} \frac{\left(\bar{s}^{4}+\bar{t}^{4}+\bar{u}^{4}\right)(\bar{u} \hat{u}+\bar{t} \hat{t})}{\bar{t} \hat{t} \bar{u} \hat{u} \bar{s} \hat{s}} \\
& \begin{aligned}
\Phi_{g g \rightarrow g g}^{(1)}= & \frac{1}{2 N_{c}^{2}}\left(N_{c}^{2} \mathscr{F}_{g g}^{(1)}-2 \mathscr{F}_{g g}^{(3)}+\mathscr{F}_{g g}^{(4)}\right. \\
& \left.+\mathscr{F}_{g g}^{(5)}+N_{c}^{2} \mathscr{F}_{g g}^{(6)}\right)
\end{aligned} \\
& K_{g g \rightarrow g g}^{(2)}=-\frac{N_{c}}{2 C_{F}} \frac{\left(\bar{s}^{4}+\bar{t}^{4}+\bar{u}^{4}\right)(\bar{u} \hat{u}+\bar{t} \hat{t}-\bar{s} \hat{s})}{\bar{t} \hat{t} \bar{u} \hat{u} \hat{s} \hat{s}} \\
& \begin{aligned}
\Phi_{g g \rightarrow g g}^{(2)}= & \frac{1}{N_{c}^{2}}\left(N_{c}^{2} \mathscr{F}_{g g}^{(2)}-2 \mathscr{F}_{g g}^{(3)}+\mathscr{F}_{g g}^{(4)}\right. \\
& \left.+\mathscr{F}_{g g}^{(5)}+N_{c}^{2} \mathscr{F}_{g g}^{(6)}\right)
\end{aligned}
\end{aligned}
$$

The Mandelstam variables with a 'hat' and a 'bar are defined as follows

$$
\begin{array}{ll}
\hat{s}=\left(k_{A}+k_{B}\right)^{2}=\left(p_{1}+p_{2}\right)^{2}, & \bar{s}=\left(x_{A} p_{A}+k_{B}\right)^{2}, \\
\hat{t}=\left(p_{2}-k_{B}\right)^{2}=\left(p_{1}-k_{A}\right)^{2}, & \bar{t}=\left(x_{A} p_{A}-p_{1}\right)^{2}, \\
\hat{u}=\left(p_{1}-k_{B}\right)^{2}=\left(p_{2}-k_{A}\right)^{2}, & \bar{u}=\left(x_{A} p_{A}-p_{2}\right)^{2},
\end{array}
$$

where $k_{A}=x_{A} p_{A}+k_{T}$ and $k_{B}=x_{B} p_{B}$ are momenta of the incoming partons, with the first one being off-shell. The matrix elements entering the TMD gluon distributions are

$$
\begin{gathered}
\mathscr{F}_{q g}^{(1)}=2 \int \frac{d \xi^{+} d^{2} \xi_{T}}{(2 \pi)^{3} p_{A}^{-}} e^{i x_{A} p_{A}^{-} \xi^{+}-i \vec{k}_{T} \cdot \vec{\xi}_{T}}\left\langle p_{A}\left|\operatorname{Tr}\left\{F(\xi) \mathscr{U}^{[-] \dagger} F(0) \mathscr{U}^{[+]}\right\}\right| p_{A}\right\rangle, \\
\mathscr{F}_{q g}^{(2)}=2 \int \frac{d \xi^{+} d^{2} \xi_{T}}{(2 \pi)^{3} p_{A}^{-}} e^{i x_{A} p_{A}^{-} \xi^{+}-i \vec{k}_{T} \cdot \vec{\xi}_{T}}\left\langle p_{A}\left|\operatorname{Tr}\left\{F(\xi) \frac{\operatorname{Tr} \mathscr{U}^{[\square]}}{N_{c}} \mathscr{U}^{[+] \dagger} F(0) \mathscr{U}^{[+]}\right\}\right| p_{A}\right\rangle, \\
\mathscr{F}_{g g}^{(1)}=2 \int \frac{d \xi^{+} d^{2} \xi_{T}}{(2 \pi)^{3} p_{A}^{-}} e^{i x_{A} p_{A}^{-} \xi^{+}-\vec{i}_{T} \cdot \vec{\xi}_{T}}\left\langle p_{A}\left|\operatorname{Tr}\left\{F(\xi) \frac{\operatorname{Tr} \mathscr{U}^{[\square]}}{N_{c}} \mathscr{U}^{[-] \dagger} F(0) \mathscr{U}^{[+]}\right\}\right| p_{A}\right\rangle, \\
\mathscr{F}_{g g}^{(2)}=2 \int \frac{d \xi^{+} d^{2} \xi_{T}}{(2 \pi)^{3} p_{A}^{-}} e^{i x_{A} p_{A}^{-} \xi^{+}-i \vec{k}_{T} \cdot \vec{\xi}_{T}} \frac{1}{N_{c}}\left\langle p_{A}\left|\operatorname{Tr}\left\{F(\xi) \mathscr{U}^{[\square] \dagger}\right\} \operatorname{Tr}\left\{F(0) \mathscr{U}^{[\square]}\right\}\right| p_{A}\right\rangle, \\
\mathscr{F}_{g g}^{(3)}=2 \int \frac{d \xi^{+} d^{2} \xi_{T}}{(2 \pi)^{3} p_{A}^{-}} e^{i x_{A} p_{A}^{-} \xi^{+}-\vec{k}_{T} \cdot \vec{\xi}_{T}}\left\langle p_{A}\left|\operatorname{Tr}\left\{F(\xi) \mathscr{U}^{[+] \dagger} F(0) \mathscr{U}^{[+]}\right\}\right| p_{A}\right\rangle, \\
\mathscr{F}_{g g}^{(4)}=2 \int \frac{d \xi^{+} d^{2} \xi_{T}}{(2 \pi)^{3} p_{A}^{-}} e^{i x_{A} p_{A}^{-} \xi^{+}-i \vec{k}_{T} \cdot \vec{\xi}_{T}}\left\langle p_{A}\left|\operatorname{Tr}\left\{F(\xi) \mathscr{U}^{[-] \dagger} F(0) \mathscr{U}^{[-]}\right\}\right| p_{A}\right\rangle,
\end{gathered}
$$




$$
\begin{aligned}
& \mathscr{F}_{g g}^{(5)}=2 \int \frac{d \xi^{+} d^{2} \xi_{T}}{(2 \pi)^{3} p_{A}^{-}} e^{i x_{A} p_{A}^{-} \xi^{+}-i \vec{k}_{T} \cdot \vec{\xi}_{T}}\left\langle p_{A}\left|\operatorname{Tr}\left\{F(\xi) \mathscr{U}^{[\square] \dagger} \mathscr{U}^{[+] \dagger} F(0) \mathscr{U}^{[\square]} \mathscr{U}^{[+]}\right\}\right| p_{A}\right\rangle, \\
& \mathscr{F}_{g g}^{(6)}=2 \int \frac{d \xi^{+} d^{2} \xi_{T}}{(2 \pi)^{3} p_{A}^{-}} e^{i x_{A} p_{A}^{-} \xi^{+}-i \vec{k}_{T} \cdot \vec{\xi}_{T}}\left\langle p_{A}\left|\operatorname{Tr}\left\{F(\xi) \mathscr{U}^{[+] \dagger} F(0) \mathscr{U}^{[+]}\right\}\left(\frac{\operatorname{Tr} \mathscr{U}^{[\square]}}{N_{c}}\right)^{2}\right| p_{A}\right\rangle,
\end{aligned}
$$

with the following definitions of gauge links and loops:

$$
\mathscr{U}^{[ \pm]}=U\left(0, \pm \infty ; 0_{T}\right) U\left( \pm \infty, \xi^{+} ; \xi_{T}\right), \quad \mathscr{U}^{[\square]}=\mathscr{U}^{[+]} \mathscr{U}^{[-] \dagger}=\mathscr{U}^{[-]} \mathscr{U}^{[+] \dagger},
$$

where $U\left(a, b ; x_{T}\right)=\mathscr{P} \exp \left[i g \int_{a}^{b} d x^{+} A_{a}^{-}\left(x^{+}, x_{T}\right) t^{a}\right]$. The two TMD gluon distributions indicated above in the blue color, that is $\mathscr{F}_{q g}^{(1)}$ and $\mathscr{F}_{g g}^{(3)}$ are the ones that turn out to be universal in the large $N_{c}$ limit and under certain CGC-motivated assumptions [14]. They build up all the other gluon distributions remaining in the large $N_{c}$ limit.

\section{Outlook}

The factorization formula (3.1) is supposed to be valid for a broad range of scales apparent in the process under consideration $Q_{s} \lesssim k_{T} \lesssim P_{T}$. Although it is not a strict theorem of perturbative QCD, it has limits which are solid results of QCD. Namely, when $k_{T} \sim Q_{s} \ll P_{T}$, i.e. in the backto-back saturation limit, the result of [14] is recovered (which in turn follows from CGC). On the other hand, when $Q_{s} \ll k_{T} \sim P_{T}$, i.e. we approach a dilute limit, the High Energy Factorization is recovered.

The next challenging step is to extract the two universal unintegrated gluon distributions from models or fits and calculate the remaining distributions needed to apply (3.1) in practice.

\section{Acknowledgments}

The author thanks A. van Hameren, K. Kutak, C. Marquet, E. Petreska and S. Sapeta for a fruitful collaboration on the subject. The author also acknowledges the support of the grants DEC-2011/01/B/ST2/03643 and DE-FG02-93ER40771.

\section{References}

[1] L. V. Gribov, E. M. Levin and M. G. Ryskin, Phys. Rept. 100 (1983) 1.

[2] A. Dumitru, A. Hayashigaki and J. Jalilian-Marian, Nucl. Phys. A 765, 464 (2006)

[3] F. Gelis, E. Iancu, J. Jalilian-Marian and R. Venugopalan, Ann. Rev. Nucl. Part. Sci. 60 (2010) 463.

[4] J. L. Albacete and C. Marquet, Prog. Part. Nucl. Phys. 76 (2014) 1.

[5] J. L. Albacete and C. Marquet, Phys. Rev. Lett. 105 (2010) 162301.

[6] A. Stasto, B. -W. Xiao and F. Yuan, Phys. Lett. B 716 (2012) 430.

[7] L. N. Lipatov, Phys. Rept. 286, 131 (1997),

[8] S. Catani, M. Ciafaloni and F. Hautmann, Nucl. Phys. B 366 (1991) 135. 
[9] J. C. Collins \& R. K. Ellis, Nucl. Phys. B360, 3 (1991).

[10] M. Deak, F. Hautmann, H. Jung and K. Kutak, JHEP 0909 (2009) 121.

[11] A. van Hameren, P. Kotko, K. Kutak and S. Sapeta, Phys. Lett. B 737, 335 (2014)

[12] P. Kotko, W. Slominski and D. Toton, arXiv:1504.00823 [hep-ph].

[13] A. van Hameren, P. Kotko and K. Kutak, arXiv:1505.02763 [hep-ph].

[14] F. Dominguez, C. Marquet, B. -W. Xiao and F. Yuan, Phys. Rev. D 83 (2011) 105005.

[15] C. J. Bomhof, P. J. Mulders and F. Pijlman, Eur. Phys. J. C 47 (2006) 147.

[16] CMS Collaboration [CMS Collaboration], CMS-PAS-FSQ-12-008.

[17] R. Aaij et al. [LHCb Collaboration], JHEP 1401, 033 (2014)

[18] P. Kotko, K. Kutak, C. Marquet, E. Petreska, S. Sapeta and A. van Hameren, arXiv:1503.03421 [hep-ph].

[19] A. van Hameren, P. Kotko and K. Kutak, JHEP 1212 (2012) 029.

[20] A. van Hameren, P. Kotko and K. Kutak, JHEP 1301 (2013) 078.

[21] P. Kotko, JHEP 1407 (2014) 128.

[22] E. N. Antonov, L. N. Lipatov, E. A. Kuraev and I. O. Cherednikov, Nucl. Phys. B 721 (2005) 111.

[23] M. L. Mangano and S. J. Parke, Phys. Rept. 200 (1991) 301. 